\title{
Video Article \\ Thin Film Composite Silicon Elastomers for Cell Culture and Skin Applications: Manufacturing and Characterization
}

\author{
Silviya Boyadzhieva* ${ }^{1,2}$, Sarah C.L. Fischer ${ }^{1,2}$, Svenja Lösch ${ }^{1,3}$, Angela Rutz ${ }^{1}$, Eduard Arzt ${ }^{1,2}$, Klaus Kruttwig ${ }^{1}$ \\ ${ }^{1}$ INM - Leibniz Institute for New Materials \\ ${ }^{2}$ Department of Materials Science and Engineering, Saarland University \\ ${ }^{3}$ University of Applied Sciences Kaiserslautern \\ * These authors contributed equally
}

Correspondence to: Klaus Kruttwig at Klaus.Kruttwig@leibniz-inm.de

URL: https://www.jove.com/video/57573

DOI: doi:10.3791/57573

Keywords: Developmental Biology, Issue 137, poly(di-methylsiloxane), thin film composite, elastomers, skin adhesives, adhesion, cell culture

Date Published: $7 / 3 / 2018$

Citation: Boyadzhieva, S., Fischer, S.C., Lösch, S., Rutz, A., Arzt, E., Kruttwig, K. Thin Film Composite Silicon Elastomers for Cell Culture and Skin Applications: Manufacturing and Characterization. J. Vis. Exp. (137), e57573, doi:10.3791/57573 (2018).

\section{Abstract}

In this protocol, we present methods to fabricate thin elastomer composite films for advanced cell culture applications and for the development of skin adhesives. Two different poly-(dimethyl siloxanes) (PDMS and soft skin adhesive (SSA)), have been used for in depth investigation of biological effects and adhesive characteristics. The composite films consist of a flexible backing layer and an adhesive top coating. Both layers have been manufactured by doctor blade application technique. In the present investigation, the adhesive behavior of the composite films has been investigated as a function of the layer thickness or a variation of the Young's modulus of the top layer. The Young's modulus of PDMS has been changed by varying the base to crosslinker mixing ratio. In addition, the thickness of SSA films has been varied from approx. $16 \mu \mathrm{m}$ to approx. $320 \mu \mathrm{m}$. Scanning electron microscopy (SEM) and optical microscopy have been used for thickness measurements. The adhesive properties of elastomer films depend strongly on the film thickness, the Young's modulus of the polymers and surface characteristics. Therefore, normal adhesion of these films on glass substrates exhibiting smooth and rough surfaces has been investigated. Pull-off stress and work of separation are dependent on the mixing ratio of silicone elastomers.

Additionally, the thickness of the soft skin adhesive placed on top of a supportive backing layer has been varied in order to produce patches for skin applications. Cytotoxicity, proliferation and cellular adhesion of L929 murine fibroblasts on PDMS films (mixing ratio 10:1) and SSA films (mixing ratio 50:50) have been conducted. We have shown here, for the first time, the side by side comparison of thin composite films manufactured of both polymers and present the investigation of their biological- and adhesive properties.

\section{Video Link}

The video component of this article can be found at https://www.jove.com/video/57573/

\section{Introduction}

In this protocol, detailed methods for manufacturing of thin elastomer films are presented. The widely available doctor blade technique has been used for the production of thin composite films. The manufacturing technique has been performed on polyethylenterephtalate (PET) foils, enabling subsequent production of these films in large scale. Emphasis of this protocol is the assessment of reproducibility, precise manufacturing of the different layers of the composite films and determination of the biological and adhesion properties of the final composite patch. The silicone elastomer poly-(dimethylsiloxane) (PDMS) is extensively used in biomedical technology, including production of skin adhesives, microfluidics applications and additional research fields ${ }^{1,2,3,4}$. Recently, another subclass of PDMS, so called Soft Skin Adhesives (SSAs) have been introduced, particular for gentle skin bonding and de-bonding

Silicone SSAs are vinyl functionalized elastomers, differing from analogous polymers by the absence of reinforcing silica ${ }^{5}$. Similar to other PDMS, SSA's Young's modulus can be adapted in a wide range by modulating cross-linker concentration or curing time ${ }^{6,7,8}$. This change in Young's modulus of silicone elastomers affects the adhesive properties of the material significantly and has also profound consequences on prokaryotic and eukaryotic cells cultured on the surface ${ }^{9,10,11}$. On the cellular biological level, it was shown, that eukaryotic cells respond on the signal transduction level to a modulation of the matrix elasticity or thickness of the surface $e^{9,10,12,13,14}$. Therefore, a broad interest in cell culture applications of polymers with tunable mechanical properties exist. Importantly, the intrinsically low surface energy of silicone based elastomers does not provide optimal conditions for cell culture of eukaryotic cells. Oxygen plasma treatment is a widely used technique to increase PDMS low surface energy temporarily, leading to an enhancement of its pull-off strength, decreased surface adsorption of molecules, while in parallel promoting attachment, spreading and proliferation of eukaryotic cells ${ }^{15,16,17,18}$.

In addition to the materials properties, the surface topography significantly affects cellular adhesion and the adhesive interaction between two materials $^{19,20,21,22}$. Surface roughness has several effects on the contact formation between two surfaces: reduction of the contact area, high 
stored elastic energy surrounding asperities as well as influence on the crack propagation can alter the adhesive strength ${ }^{23,24}$. Adhesion of selfadhesive films to human skin is an emerging application field, e.g., wound dressings, fixation of ECG electrodes or other wearable electronic devices $^{25,26,27,28}$. To measure the adhesive performance of self-adhesives in relation to surface topography, glass substrates with varying degrees of roughness can be used in normal adhesion measurements ${ }^{8,21}$. Here, two glass substrates have been selected to investigate the adhesive properties of the polymer films. First, composite films with a PDMS backing layer in a mixing ratio of 10 to 1 weight parts covered by PDMS with different mixing ratio were characterized. In a second step a adhesive SSA layer was prepared with equal weight amounts of both components and with varying film thickness on top of a supporting PDMS film.

\section{Protocol}

Caution: Please consult all relevant material safety data sheets (MSDS) before use. Some of the chemicals used in this protocol are irritants, acutely toxic and/or carcinogenic. Please use all appropriate safety practices when handling these chemicals. This includes the use of engineering (chemical cabinet) and personal protective equipment (safety glasses, gloves, lab coat, full length pants and closed-toe shoes). Portions of the following procedures involve the culture of an animal cell line. Therefore, please follow the specific biosafety regulations. Chemical and biological waste needs to be disposed according to the specific national and institutional rules and recommendations.

\section{Preparation of Silicon Elastomeric Thin Film Composite Structures}

1. Preparation of polymers

1. To prepare $1.1 \mathrm{~g}$ of PDMS in the ratio $10: 1$, mix $1.0 \mathrm{~g}$ of compound $\mathrm{A}$ with $0.1 \mathrm{~g}$ of compound $\mathrm{B}$.

2. Mix and degase the pre-polymers in a speed mixer at $2350 \mathrm{rpm}$ under vacuum for $3 \mathrm{~min}$.

3. Change the mass ratios between compound $A$ and compound $B$ to $45: 1$ and 70:1. Prepare them similar to the method described in 1.1.2.

4. Prepare $1 \mathrm{~g}$ of the soft skin adhesive (SSA) in the ratio of 50:50. Therefore mix $0.5 \mathrm{~g}$ of compound $\mathrm{A}$ and $0.5 \mathrm{~g}$ of compound $\mathrm{B}$ as described in 1.1.2.

2. Preparation of poly-(vinyl alcohol) (PVA) coated PET foil

1. Prepare a $18 \%(\mathrm{w} / \mathrm{w})$ PVA solution in water by adding PVA to deionized water and mix overnight with a magnetic stirrer. Store this solution at $4{ }^{\circ} \mathrm{C}$.

2. Prepare thin films exhibiting an effective thickness of $15 \mu \mathrm{m}$ with the doctor blade application machine, using $100 \mu \mathrm{m}$ gap of the blade and a velocity of approx. $2.0 \mathrm{~mm} / \mathrm{s}$.

3. Place the films in an oven at $95^{\circ} \mathrm{C}$ for $15 \mathrm{~min}$

3. Preparation of the backing-layer of PDMS 10:1 mixing ratio by doctor blade technique

1. Use an automatically controlled doctor blade application machine for the preparation of the thin films.

2. Clean the PET foil with $100 \%$ isopropanol and place it on the surface of the doctor blade application area.

3. Place the doctor blade on top of the foil and adjust the thickness with the micro positioning screws. For manufacturing of the wet layers, apply thicknesses of $60 \mu \mathrm{m}, 100 \mu \mathrm{m}, 200 \mu \mathrm{m}$ and $500 \mu \mathrm{m}$.

4. Fill the PDMS 10:1 polymer prepared in step 1.1 into the reservoir of the doctor blade with a single use syringe. Start movement of the blade with a velocity of approx. $2.0 \mathrm{~mm} / \mathrm{s}$.

5. Remove the PET film with the applied 10:1 coating from the machine and place it in an oven for $1 \mathrm{~h}$ at $95{ }^{\circ} \mathrm{C}$, located in a room exhibiting humidity between $40 \%$ and $65 \%$.

6. Clean the doctor blade with isopropanol and paper towels.

7. Repeat this procedure for all required thicknesses.

4. Preparation of the top layer of PDMS in different mixing ratios by doctor blade technique

1. Remove thin stripes of the length sides of the underlying film with a scalpel or razor blade to allow placement and sliding of the doctor blade on the PET foil.

2. Follow protocol steps 1.3.3 to 1.3.6. Wet thickness applied for the film is $160 \mu \mathrm{m}$.

3. Repeat this procedure for the production of two independent films each with another mixing ratio of the PDMS components (45:1 and $70: 1$ ). Store the films at room temperature (approx. $22^{\circ} \mathrm{C}$ and at a humidity between 40 and $65 \%$ ) in square Petri dishes to prevent them from contamination and dust.

5. Preparation of thin composite films exhibiting different thicknesses of the SSA 50:50 layer

1. Prepare PDMS 10:1 films as a backing-layer, as described before in step 1.3 .

2. Follow protocol steps 1.4.1 and 1.4.2 to produce these films. Use SSA in the mixing ratio of 50:50 and manufacture a film with a wet thickness of $40 \mu \mathrm{m}$.

3. Repeat the procedure for the additional wet thicknesses of: $120 \mu \mathrm{m}, 300 \mu \mathrm{m}, 500 \mu \mathrm{m}$.

\section{Normal Adhesion Measurements Using Substrates with Different Surface Roughness}

1. Preparation and characterization of glass substrates with different surface roughness

1. Use a glass cylinder with $2 \mathrm{~mm}$ diameter as a 'smooth substrate'.

2. To manufacture the 'rough substrate' excise with a glass cutter a piece with the dimension of about $4 \times 4 \mathrm{~mm}$ from a frosted glass slide. Use an abrasive diamond hand pad to obtain a circular area of approx. $3 \mathrm{~mm}$ diameter.

3. Attach the glass to an aluminum cone with UV glue and illuminate it in the UV illumination chamber for $3 \mathrm{~min}$. 
4. Determine the radius of the surface area of the substrate with an optical microscope. Calculate the area according to the formula $A=\pi r^{2}$.

5. Determine the roughness parameter $R_{a}$ and $R_{z}$ (according to: DIN EN ISO 4287, ASME B46.1) with a stylus profilometer.

6. Affix the substrate on the sample stage of the profilometer and bring the tip (diamond, standard: $2 \mu \mathrm{m} / 60^{\circ}$ ) into contact with the sample.

7. Record the roughness profile with a velocity of $0.3 \mathrm{~mm} / \mathrm{s}$ and a length of $1 \mathrm{~mm}$.

8. To analyze the surface topography, measure an area of exactly $1 \mathrm{~mm}^{2}$ with a stylus profilometer, operated by the associated software. NOTE: The profilometer is operated by an external computer. The holder is shifted by $0.001 \mathrm{~mm}$ in y direction after a displacement of 1 $\mathrm{mm}$ is reached in $\mathrm{x}$ direction. The recorded .RS3 file is imported in Surfcom Map Expert Software to create 3D images.

2. Normal adhesion measurement of thin films manufactured of PDMS or SSA

1. Use a razor blade to cut the films on the PET foil into small pieces with an area of about $4.0 \mathrm{~cm}^{2}$ and place them on a glass slide with UV glue. Illuminate with UV light for 3 min.

2. Mount the polymeric sample on the sample holder.

3. Clean the substrate surface gently with ethanol and dry with nitrogen gas.

4. Attach the glass substrate, mounted to the aluminum cone, to the load cell.

5. Use the tiltable table (goniometer) to align the surfaces precisely by adjusting the tilt angle of the substrate approaching the polymeric film. In order to do this, bring the substrate manually in contact with the film. Change the tilt angle until a completely parallel alignment of both surfaces to each other, visualized by the cameras images, is obtained.

NOTE: The load cell is connected to the tiltable table. A glass prism is located below the sample as shown in Figure 4, allowing visualization of the contact area with two cameras and enabling the alignment of the substrate on the polymer film.

6. Move the substrate to the polymeric film surface until a preload stress of $13 \pm 5 \mathrm{kPa}$ is achieved (Figure 4).

7. Start the custom programmed software package written in LabView to control the required measurement parameters such as hold time and approach/retraction velocity. The hold time $t_{\text {hold }}$ is 1 second, the approach and detachment velocity is $30 \mu \mathrm{m} / \mathrm{s}$ and $10 \mu \mathrm{m} / \mathrm{s}$ respectively.

8. Perform adhesion measurements on three independent manufactured samples and at six different locations on each film surface.

3. Data analysis and calculation of key mechanical factors: pull-off stress and work of separation.

1. Calculate the stress $\sigma$ by dividing the recorded force by the substrate area $\mathrm{A}_{\mathrm{S}}$.<smiles>[14CH3][14C]([14CH3])=O</smiles>

2. Determine the pull-off stress, which is described as the maximum value of the normal stress.

$\sigma_{\max }=\max \left(\frac{F}{A_{s}}\right)$

3. Obtain the displacement $\Delta \mathrm{s}$ by subtraction of the start position of the tensile regime $\mathrm{s}_{\mathrm{s}}$ from the sample position $\mathrm{s}_{\text {end }}$ where de-bonding has been completed. Define the start of the tensile regime as $\mathrm{s}_{0}=0$.

$\Delta S=s_{\text {end }}-S_{0}$

4. Correct the measured values of the sample position by the system compliance $\mathrm{C}$ according to the following equation:

$s=s_{\text {measured }}-C * F$

5. Integrate the stress-displacement curve between $\mathrm{s}_{0}$ and $\mathrm{s}_{\mathrm{end}}$ in order to calculate the work of separation.

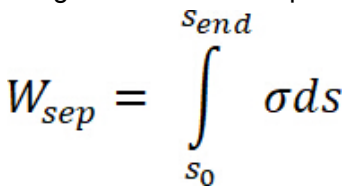

4. Calculation of key mechanical factors using mathematical computing software Origin.

1. Import the recorded dat file from a single adhesion measurement in an origin table. The parameters that are recorded are time, sample position and force. Insert these parameters in the columns A (time), B (sample position) and C (force).

2. To determine the blank value, average about 20 measurement values of the force before contacting the polymer film. Name this average value $F_{\text {offset }}$ and paste it in column $D$.

3. Calculate the background corrected force $\mathrm{F}^{\star}$ according to the following equation

$F^{*}=F-F_{\text {offset }}$

and insert this equation, as shown below, into column $\mathrm{E}$.

$\operatorname{col}(E)=\operatorname{col}(C)-F_{\text {offset }}$

4. Define the start of the tensile regime as zero displacement, i.e., $\mathrm{s}_{0}=0$. Therefore, determine $\mathrm{s}_{0}$ and subtract it from the displacement in Column $\mathrm{B}$ and save it in Column $\mathrm{F}$ :

$\operatorname{col}(F)=\operatorname{col}(B)-s_{0}$

5. Furthermore, correct the sample position by the machine compliance. This correction is performed in column $\mathrm{G}$. Insert the following equation into column $\mathrm{G}$

$\operatorname{col}(G)=\operatorname{col}(F)-C * \operatorname{col}(E)$

6. Calculate the stress in the next column $\mathrm{H}$. Therefore, divide the force by the substrate area. Insert the following equation 
$\operatorname{col}(H)=\frac{\operatorname{col}(E)}{A}$

where $A$ is the surface area of the glass substrates in $\mathrm{mm}^{2}$ (determined in 2.1).

7. Calculate the work of separation from the stress and displacement values. Therefore, plot the displacement along the $x$-axis and the stress along the $y$-axis. Integrate this graph from $s_{0}$ to $s_{e n d}$ where $s_{\text {end }}$ is defined as the displacement at which tensile stress returns to zero, i.e. full detachment took place. To integrate the graph, choose the integrate function. Add the calculated values into columns I and $\mathrm{J}$.

\section{Characterization of the Films by Scanning Electron Microscopy (SEM) and Optical Microscopy}

\section{Optical microscopy}

1. With a razor blade cut the polymeric film into small pieces (approx. $0.25 \mathrm{~cm}^{2}$ ) and attach them to the edge of a glass slide. Place the glass slide vertically orientated under an upright microscope and measure the thickness of the film cross-section. NOTE: Use a 20X objective (NA $=0.45$, theoretical resolution at $800 \mathrm{~nm}$ of $1.1 \mu \mathrm{m}$ ) to measure film thickness values of approx. $\leq 20$ $\mu \mathrm{m}$. For a film thickness in the range of $20 \mu \mathrm{m}$ up to $50 \mu \mathrm{m}$ use a 10X objective (NA $=0.30$, theoretical resolution at $800 \mathrm{~nm}$ of $1.6 \mu \mathrm{m}$ ) and for a film thickness $\geq 50 \mu \mathrm{m}$ use a $5 \mathrm{X}$ objective (NA $=0.15$, theoretical resolution at $800 \mathrm{~nm}$ of $3.3 \mu \mathrm{m})$.

\section{SEM investigation}

1. Cut the PET foil and attach a sample of approx. $2 \mathrm{~cm}^{2}$ to a glass slide and place it vertically to the clamping mechanism inside the sample holder $\leq 2 \mathrm{~mm}$ below the top surface of the holder.

2. Select an acceleration voltage of $10 \mathrm{kV}$, the backscattered electron detector (BSD) and low vacuum conditions (60 Pa).

3. Adjust the focus, magnification, brightness and contrast of the images.

4. Choose an image acquisition time of $28 s$ with a resolution of $1024 \times 2048$ pixels.

5. Remove the sample holder from the SEM.

\section{Biological Investigation}

\section{Routine cell Culture of L929 cells}

1. Use the murine fibroblast cell line L929 for investigation. Culture the cells in Rosewell Park Memorial Institute (RPMI) 1640 basal medium, supplemented with $10 \%$ fetal bovine serum and penicillin und streptomycin at $37{ }^{\circ} \mathrm{C}, 5 \% \mathrm{CO}_{2}$ in T75 cell culture flasks. Passage the cells at a confluence of approximately $70 \%$ to $80 \%$.

2. For cell passaging, remove the medium by aspiration and wash with calcium- and magnesium free phosphate buffer (DPBS ${ }^{-/-}$) for $30 \mathrm{~s}$ under a laminar flow cabinet. Afterwards incubate the cells with $2 \mathrm{~mL}$ of Accutase, an enzyme solution with proteolytic and collagenolytic activity for up to $5 \mathrm{~min}$ at $37^{\circ} \mathrm{C}, 5 \% \mathrm{CO}_{2}$

3. Verify detachment of the cells from the cell culture flask surface with a phase contrast microscope.

4. Add $8 \mathrm{~mL}$ of serum-containing medium into the flask and transfer the cell suspension to a $15 \mathrm{~mL}$ reaction tube.

5. Take a $10 \mu \mathrm{L}$ sample from the cell suspension and mix with $10 \mu \mathrm{L}$ of Trypan Blue.

6. Determine the cellular number with a Neubauer chamber and calculate the total number of cells. CAUTION: Trypan Blue is toxic, therefore consulting the MSDS, following the mandatory procedures described in the MSDS, wearing of appropriate personal safety protection and handling under a chemical cabinet is required. Collect waste for chemical waste deposition.

NOTE: Trypan Blue positive cells are colored blue, indicating non-intact cellular membranes.

7. For the next passage, culture $5 \times 10^{5}$ cells in a new sterile cell culture flask with $10 \mathrm{~mL}$ of new medium. For experimental conditions, culture $3 \times 10^{5}$ cells in the 6 well plates and $6 \times 10^{4}$ cells in each well of the 24 well plate, containing polymeric samples (protocol step 4.2).

2. Preparation of composite films for cell culture experiments.

1. Excise single pieces of films of the desired dimensions manufactured in protocol step 1.4 and 1.5 from the PET supportive layer with a scalpel and place with tweezers onto the surface of glass cover slips exhibiting a diameter of $12 \mathrm{~mm}$. Place the samples in the wells of a 24 well plate.

2. For cytotoxicity determination and cell counting, do not remove the films from the PET foil. Cut circular areas of approx. $9.4 \mathrm{~cm}^{2}$, fitting neatly into the single wells of a 6 well plate, from the films produced in 1.4 and 1.5 and place them into the wells of a cell culture plate.

3. Immerse the polymer samples in deionized $\mathrm{H}_{2} \mathrm{O}$ for $\geq 30$ min.

NOTE: The polymeric samples might be sterilized by autoclaving. Therefore, remove all polymer containing samples from the cell culture dishes and place them inside a glass Petri dish. Sterilization is performed in an autoclave at 2.05 bar for 20 min at a temperature of $121^{\circ} \mathrm{C}$.

\section{Plasma treatment of polymers}

1. Place the films which are attached on PET foil or on round glass cover slips (manufactured in 4.2.1) inside the reaction chamber of the plasma device.

2. Close the lid and evacuate until a pressure of $1.6 \times 10^{-2}$ mbar is reached.

3. Perform plasma treatment for 3 min.

4. Ventilate the reaction chamber and place samples in either 24 well or 6 well dishes for further cell culture investigations. 
5. Use one sample for water contact angle determination with a goniometer. Therefore, move the syringe close to the polymeric surface, using the software package and place a drop of $3 \mu \mathrm{L}$ water on top of the surface. Calculate the static water contact angle with the goniometer software.

4. Staining and microscopy

1. Prepare cells as described in step 4.1 .7 and culture for $3 \mathrm{~d}$ at $37{ }^{\circ} \mathrm{C}$ and $5 \% \mathrm{CO}_{2}$.

2. Capture phase contrast pictures of cells cultured for three days on pristine- and plasma treated films shortly before fixation.

3. Prepare PBS supplemented with $0.2 \%$ Triton-X-100. Slowly pipette $200 \mu \mathrm{L}$ of the stock solution into $100 \mathrm{~mL}$ of PBS (PBS-T).

4. Prepare a $4 \%$ paraformaldehyde/PBS solution (PFA/PBS-T).

CAUTION: Paraformaldehyde is toxic, therefore consulting the MSDS, following the mandatory procedures described in the MSDS and wearing of appropriate personal safety protection and handling under a chemical cabinet is required.

5. Prepare a $5 \%$ BSA/PBS-T solution.

6. Remove the medium by aspiration under the lamina flow cabinet. Add PBS to the wells to remove medium residues.

7. Transfer the plate to a chemical cabinet and replace PBS with $400 \mu \mathrm{L}$ of the PFA/PBS solution for 25 minutes at room temperature.

8. Remove the PFA/PBS solution from the single wells, carefully wash with PBS four times. Wait 3 min. between each washing step and collect the solutions for chemical waste disposal. Use the plate directly, or store it at $4{ }^{\circ} \mathrm{C}$.

9. Add $5 \%$ bovine serum albumin (BSA)/PBS-T to the wells and incubate for 60 min at RT to block unspecific binding sites.

10. Aspirate the solution and replace it with a phalloidin conjugated to Alexa-488 (1:160 dilution)/PBS-T solution supplemented with $0.2 \%$ Triton-X-100.

CAUTION: Phalloidin-488 is toxic, therefore consulting the MSDS, following the mandatory procedures described in the MSDS and wearing of appropriate personal safety protection and handling under a chemical cabinet is required.

11. Cover the plate with aluminum foil and incubate it for $3 \mathrm{~h}$ at RT or overnight at $4{ }^{\circ} \mathrm{C}$.

12. Aspirate the solution and wash three times with PBS. Wait $3 \mathrm{~min}$. between each washing step. Collect the solutions for chemical waste disposal.

13. Prepare a solution of $1 \mu \mathrm{L}$ of Hoechst Dye 33342 (stock solution $1 \mathrm{mg} / \mathrm{mL}$ ). For a 1:1000 dilution pipette $1 \mu \mathrm{L}$ of Hoechst Dye 3334 to 1 $\mathrm{mL}$ of PBS-T and mix well. Add $300 \mu \mathrm{L}$ of the Hoechst dye 33342 solution to the wells and incubate for $10 \mathrm{~min}$ at RT in the dark. CAUTION: Hoechst Dye 33342 is a DNA intercalating reagent and therefore potentially mutagenic, therefore consulting the MSDS, following the mandatory procedures described in the MSDS and wearing of appropriate personal safety protection and handling under a chemical cabinet is required.

14. Aspirate the solution and wash the samples four times with PBS. Wait 3 min between each washing step. Collect the solution for chemical waste disposal.

15. For embedding, carefully remove the films from the culture surface and place them on a microscope glass slide. Add 20 to $40 \mu \mathrm{L}$ of water soluble embedding medium to the film and attach a new circular glass cover slip on top using slight pressure.

16. Perform imaging with a fluorescence microscope. Filters needed for illumination: Alexa-488 has an excitation maximum at $496 \mathrm{~nm}$ and maximum emission occurs at $519 \mathrm{~nm}$. Therefore, the emission color is green. Hoechst dye 33342 trihydrochlorid trihydrate complexed with DNA has an excitation maximum at $355 \mathrm{~nm}$ and maximum DNA complex emission occurs at $465 \mathrm{~nm}$.

\section{Cytotoxicity determination and determination of cell number}

1. Perform the experiment with cells grown in the 6 well plates prepared in step 4.3 and cells prepared in step 4.1.7. Culture the cells for 3 days at $37{ }^{\circ} \mathrm{C}$ and $5 \% \mathrm{CO}_{2}$. For the positive control, use cells that were grown on a cell culture treated polystyrene surface, containing no polymeric films. For background determination (negative condition), obtain medium from a well without cells. NOTE: Medium can also be taken from the well containing cells cultured on the cell culture treated polystyrene surface.

2. According to the number of experimental samples label $15 \mathrm{~mL}$ tubes.

3. Add $40 \mu \mathrm{L}$ of a $0.9 \%$ Triton $\mathrm{X}-100$ containing PBS solution to the positive control and mix vigorously with a $1000 \mu \mathrm{L}$ tip. Wait for approx. 3 min.

4. Without removing the cells attached to the surface, aspirate the medium from all samples, including samples prepared in 4.5 .3 and transfer the medium to the $15 \mathrm{~mL}$ tubes. Add $3 \mathrm{~mL}$ of DPBS ${ }^{-/-}$to the single wells and store the plates under the laminar flow cabinet for determination of the cellular number as described in 4.5.9.

5. Centrifuge at $200 \times \mathrm{g}$ for 3 minutes and remove $1 \mathrm{~mL}$ of the supernatant for the LDH activity determination. Store the $15 \mathrm{~mL}$ tubes containing cells and remaining medium under the laminar flow cabinet.

6. For the assay a black 96 well plates with flat bottom are used. Add $50 \mu \mathrm{L}$ of CytoTox-ONE Reagent to $50 \mu \mathrm{L}$ of the sample medium and mix well for $30 \mathrm{~s}$.

7. Cover the plate with aluminum foil and store for $10 \mathrm{~min}$ at RT.

8. Add $25 \mu \mathrm{L}$ of the stop solution to each well and record the fluorescence intensity with a fluorescence plate reader. Shake the plate for $10 \mathrm{~s}$ and detect the fluorescence signal with an excitation wavelength of $520 \mathrm{~nm}$ and an emission wavelength of $560 \mathrm{~nm}$. Avoid air bubbles.

9. For determination of the cell number aspirate the $\mathrm{DPBS}^{-/-}$from the wells of the culture plate from protocol step 4.5 .4 and add the solutions to the $15 \mathrm{~mL}$ reaction tubes containing the supernatants collected in protocol step number 4.5.5.

10. Centrifuge the $15 \mathrm{~mL}$ reaction tube at $200 \mathrm{xg}$ for $3 \mathrm{~min}$ and aspirate supernatant. Add $0.5 \mathrm{~mL}$ of Trypsin/EDTA and incubate for $10 \mathrm{~min}$ at $37^{\circ} \mathrm{C}$.

11. Add $2 \mathrm{~mL}$ of Trypsin/EDTA at to the wells of the plate and incubate for approx. $10 \mathrm{~min}$ at $37^{\circ} \mathrm{C}$ to detach cells from the polymeric films.

12. The cell suspension is transferred to the $15 \mathrm{~mL}$ reaction tube from step number 4.6.10. Additionally, wash the plates vigorously with serum containing medium.

13. Centrifuge the samples at $200 \times \mathrm{g}$ for $3 \mathrm{~min}$, aspirate the supernatant and add serum containing medium to the tube.

14. Determine the cellular number as described in steps 4.1 .5 and 4.1 .6 . 


\section{Representative Results}

In the first experiments, PDMS films with varying thickness and constant mixing ratio of 10:1 have been manufactured on PET films (Figure 1). Because the thickness of the backing layer can significantly influence the stiffness and handling properties of the entire composite films, in the initial experiments single films between $13 \pm 2 \mu \mathrm{m}$ and $296 \pm 13 \mu \mathrm{m}$ were manufactured (Figure 1). It is well known, that during the curing process shrinkage of the polymer films occurs. For the thinnest films, we observed a difference of $78 \% \pm 3.1 \%$ between wet and cured conditions. For the thickest films, shrinkage of $40.9 \% \pm 2.6 \%$ has been detected (Figure 1).

For the applications presented in this protocol, films need to be manually removed from the PET foil. We recognized that especially thin films are difficult to handle with forceps and are often destroyed during this process. Therefore, we investigated the influence of a thin poly-(vinyl alcohol) coating as a supportive layer. PVA possesses a high stiffness and can be easily removed due to its water solubility in downstream applications. The applied PVA coating has a thickness of approx. $17 \mu \mathrm{m}$ and therefore PDMS films coated on top of this layer are slightly thinner compared to films without the PVA coating (data not shown). Especially focusing on the handling properties, we conclude, that only the thinnest film requires a supportive PVA film for removal from the PET foil.

An effective film thickness of about $40 \mu \mathrm{m}$ was selected for all further experiments. For the production of composite films, the mixing ratio of PDMS was varied from 10:1 to 45:1 and to 70:1 and applied on top of the previously polymerized PDMS film with the doctor blade technique (Figure 2A). With the exception of the 10:1 ratio, the different films could be clearly distinguished by optical microscopy with appropriate precision. For the microscopic analysis the films were cut with a scalpel and attached to the edge of a glass slide. The higher mixing ratios of the top layer appeared visually brighter on the microscopic images compared to the 10:1 ratio of the backing layer (Figure 2B). In addition, scanning electron microscopy was used to image samples at a magnification of about $860 \mathrm{X}$ (Figure $\mathbf{2 C}$ ). A clearly observable difference in brightness between the two PDMS films, manufactured in higher mixing ratios was recognized, in contrast to the $10: 1$ ratio. The cutting procedure leaves marks, visible in the SEM pictures (Figure 2B). Based on these results, the average overall thickness of the composite films was $112 \mu \mathrm{m} \pm 5.0$ $\mu \mathrm{m}$ (Figure 2D).

In further experiments the adhesion properties of these films have been determined with normal force adhesion measurements using two different glass substrates (Figure 3). The 'smooth substrate' possesses a surface texture with an arithmetical mean roughness $R_{a}$ of $0.013 \pm$ $0.0002 \mu \mathrm{m}$ and a mean peak-to-valley $R_{z}$ of $0.12 \pm 0.004 \mu \mathrm{m}$ (Figure 3A). Substrate 2 (GS2, designated as rough) exhibited roughness values of $0.338 \pm 0.021\left(R_{a}\right) \mu \mathrm{m}$ and $2.055 \pm 0.017 \mu \mathrm{m}\left(R_{z}\right)$ (Figure 3B). With the mean radius obtained in 2.1.4 the surface area of the 'smooth' substrate was $3.2 \mathrm{~mm}^{2}$ while for the 'rough' substrate a surface area of $6.07 \mathrm{~mm}^{2}$ has been calculated.

With these two substrates, the adhesive behavior of the different films has been determined. Two parameters are chosen to describe the adhesive properties of the films: the pull-off stress $\sigma_{\max }$ and the work of separation $\mathrm{W}_{\text {sep. }}$. During the whole process of bonding and de-bonding the sample position $\mathrm{s}$ and the normal force $\mathrm{F}$ are recorded. The results are represented in a stress- displacement curve (Figure 4).

For the correct interpretation of the experimental results, it is of importance to accurately align the substrate to the polymeric film surface. Also, the machine compliance of the measurement device must to be considered in order to correct the displacement. During the measurement the applied force acts not solely on the sample, but also on other parts of the testing device. Therefore, each of the two substrates is pressed against a glass slide with a compressive stress of $13 \pm 5 \mathrm{kPa}$. To measure the compliance, the load curve is taken into account, i.e., the part of the forcedisplacement curve where the two surfaces come into contact up to the sample position where the exact preload force is reached. The reciprocal slope of the curve is equal to the machine compliance $C$. The calculated value for $C$ is $0.12 \mu \mathrm{m} / \mathrm{mN}$.

In the first experiment films with different mixing ratios of PDMS were analyzed (Figure 5). For the composite films, the thickness and the mixing ratio of the backing layer, manufactured of PDMS 10:1 was kept constant. The thickness of the top layer was also kept constant with a value of $65 \mu \mathrm{m}$. The highest pull-off stress of $109 \pm 27.6 \mathrm{kPa}$ was determined with the smooth glass substrate on the PDMS 10:1 film (Figure 5A). An increase of the mixing ratio leads to a decrease of the pull-off stress to $76.7 \pm 17 \mathrm{kPa}$ for $45: 1$ mixing ratio and $41.4 \pm 17 \mathrm{kPa}$ for the $70: 1$ ratio. With the rough glass substrate a pull-off stress of $22 \pm 2.2 \mathrm{kPa}$ was determined on the PDMS 10:1 film. In general, the work of separation was comparable between both glass substrates, e.g., $1.4 \pm 0.6 \mathrm{~J} / \mathrm{m}^{2}$ on the thinnest film obtained with the smooth substrate and $1.84 \pm 0.7 \mathrm{~J} / \mathrm{m}^{2}$ on the thinnest film obtained with the rough substrate (Figure 5B).

Next, the production of thin films for skin applications and for cell culture applications have been explored (Figure 6). SSA 50:50 has been used for the top layer production of the composite films. PDMS in a 1:10 mixing ratio with a thickness of approx. $40 \mu \mathrm{m}$ has been used as a backing layer. In contrast to the previous experiments depicted in Figure 5, the thickness of the top layer was varied, while the mixing ratio was kept constant (Figure 6A). SSA has been selected because of its adhesive properties in applications involving attachment to surfaces with high surface roughness, especially human skin, using the manufacturers recommendation of the mixing ratio $50: 50^{5,8}$. Human epidermis possesses a high surface roughness. Depending on age and anatomical region a mean surface roughness depth $\left(R_{Z}\right)$ between $48 \mu \mathrm{m}$ and $71 \mu \mathrm{m}$ has been reported $^{29}$. Secure and gentle skin adhesion is important, particularity for the sensitive skin of neonates or hardly regenerating skin of the elderly. Different wet thicknesses ranging from $40 \mu \mathrm{m}, 120 \mu \mathrm{m}, 300 \mu \mathrm{m}$ to $500 \mu \mathrm{m}$ were applied (Figure 6A). Depending on the wet thickness, the total thickness of the composite films varies between $51 \mu \mathrm{m}$ and $344 \mu \mathrm{m}$ (Figure 6B). After curing, the composite have been attached to the back of a volunteer's hand (Figure 6C). The different films thicknesses show clearly differences in their adaptation properties to the roughness of the skin (Figure 6C). Thin films (50 $\mu \mathrm{m}$ and $100 \mu \mathrm{m}$ total thickness) display a high rate of adaptation to the skin wrinkles compared to the thicker films ( $220 \mu \mathrm{m}$ and $340 \mu \mathrm{m}$ total thickness). These results indicate that composite films with a wide range of thicknesses can be produced precisely with the applied doctor blade technique. 
Adhesion experiments were performed with these composite films (Figure 7). Depending on the thickness of the SSA top film, we have observed a decrease of the pull-off stress with increasing film thickness. The highest pull-off force of $133 \pm 36.6 \mathrm{kPa}$ was measured on the smooth substrate (Figure 7A). The lowest pull-off-stress of $18 \pm 4 \mathrm{kPa}$ was obtained with the rough substrate on the thickest film. Interestingly a comparison between both substrates reveals a 2.7 fold difference on the thinnest films (Figure 7A). With increasing film thickness, especially on the thickest films no remarkable difference was observable (Figure 7A). With the smooth substrate a work of separation of $1.8 \pm 0.8 \mathrm{~J} / \mathrm{m}^{2}$ was detected on the film exhibiting a total thickness of approx. $100 \mu \mathrm{m}$, followed by a film thickness dependent decrease (220 $\mu \mathrm{m}$ thickness: 1.6 $\pm 0.6 \mathrm{~J} / \mathrm{m}^{2}$ and $330 \mu \mathrm{m}: 1.3 \pm 0.4 \mathrm{~J} / \mathrm{m}^{2}$ (Figure 7B)). The work of separation measured with the rough substrates was in general slightly lower compared to the smooth substrate $\left(100 \mu \mathrm{m}\right.$ thickness: $1.63 \pm 0.6 \mathrm{~J} / \mathrm{m}^{2} ; 220 \mu \mathrm{m}$ thickness: $1.1 \pm 0.6 \mathrm{~J} / \mathrm{m}^{2}$ and $330 \mu \mathrm{m}: 1.0 \pm 0.2 \mathrm{~J} / \mathrm{m}^{2}($ Figure 7B)).

Additionally, the detachment mechanism was recorded during the measurements (Figure 7C). Little cavitation was observed on the thinnest film, while the appearance of finger like cracks was observable on the thicker films (Figure 7C).

Measurements have been performed within one month after manufacturing of the films. However, stability and preservation of the mechanical properties of the elastic films might be impacted by environmental factors, including temperature and humidity. As described in protocol step 1.4 .3 , the films have been stored at room temperature and a humidity of $40-65 \%$. To prevent them from contamination and dust, the films have been stored in plastic Petri dishes in the dark. To investigate the long-term stability, adhesion measurements and thickness determination of SSA 50:50 films have been performed approx. four months after fabrication. No major influence on the film thickness, pull-off stress and work of separation has been detected after storage. For example, pull-off stress of the SSA composite films manufactured with a wet thickness of 120 $\mu \mathrm{m}$ SSA and a wet thickness of $100 \mu \mathrm{m}$ PDMS was $46.6 \pm 6 \mathrm{kPa}$ and the work of separation $1627 \pm 592 \mathrm{~mJ} / \mathrm{m}^{2}$ after fabrication. Approx. four months after manufacturing, a pull-off stress of $48.8 \pm 5.4 \mathrm{kPa}$ and a work of separation of $1666 \pm 723 \mathrm{~mJ} / \mathrm{m}^{2}$ was determined. In addition, shortly after manufacturing, the total thickness of these films was $103.3 \pm 13.9 \mu \mathrm{m}$ and after storage $98.1 \pm 9.1 \mu \mathrm{m}$.

In further experiments PDMS 10:1 and SSA 50:50 composite films with a total thickness of approx. $105 \mu \mathrm{m}$ have been used as cell culture substrates (Figure 8). Composite films manufactured in protocol step number 1 can be easily removed from the PET foil and cut in required dimensions and geometrical forms. Moreover, when adhering the films to a rigid surface, for example glass, multiple films displaying different Young's moduli can be attached side by side and might be placed inside a single well of a cell culture plate. Films might be attached to the polystyrene surface directly without an additional coverslip. Also, films could be adapted to different surfaces and geometrical structure, like tubes or rings, enabling further studies not achievable with conventional cell culture materials. In the performed experiments depicted in Figure 8 composite films on PET foil have been placed directly into cell culture plates or films have been removed from the PET foil and placed on glass cover slips. For the experimental conditions, some polymers have been treated with air plasma to increase their free surface energy. In general, PDMS possesses a water contact angle of approx. $115^{\circ}$ before plasma treatment and becomes highly hydrophilic (water contact angle $<30^{\circ}$ ) aftertreatment ${ }^{8}$. Plasma treatment renders the surface biocompatible and facilitates the attachment of eukaryotic cells. Depending on treatment time and intensity the polymer surface is altered, displaying a higher degree of roughness and also crack might appear. Immediately after the treatment, a hydrophobic recovery process is observed. As described under protocol step 4.3.5 a goniometer was used to determine the static water contact angles. Therefore, polymers that have been placed in $\mathrm{ddH}_{2} \mathrm{O}$ for $1 \mathrm{~h}$ after the air plasma treatment were subsequently analyzed. Plasma treatment reduced the water contact angle significantly (PDMS pristine: $117.0 \pm 2.2^{\circ}$; SSA pristine: $127.9 \pm 5.6^{\circ}$; PDMS plasma: $18.0 \pm$ $7.2^{\circ}$; SSA plasma: $29.3 \pm 11.5^{\circ}$.

For sample embedding an aqueous mounting medium has been applied. If at any time point the samples need to be removed again, the specimens can be placed in a water containing Petri dish overnight. Eventually, the cover slips can be removed for additional analysis.

The attachment behavior and morphology of L929 cells seeded for 3 days on PDMS and SSA 50:50 composite films has been determined by phase contrast microscopy and after staining with fluorescence conjugated phalloidin-488 and Hoechst dye 33342 (Figure 8). Image acquisition with phase contrast microscopy is highly recommended, especially for polymers not treated with plasma. Due to the weak cellular adhesion to these polymeric surfaces single cells or aggregates are easily detached, complicating correct interpretation of subsequent analysis methods.

Cells seeded on the pristine polymers displayed poor attachment and cellular spreading behavior (Figure 8A1 and C1) while a confluent monolayer was observed for cells cultured on plasma treated surfaces (Figure 8B1 and D1). The formation of cellular aggregates and detachment from the surface was more pronounced on pristine surfaces. Visualization of actin filaments after fixation with $4 \%$ paraformaldehyde revealed few cells migrating into the periphery of the cellular aggregates and emanation of lamellipodia protrusions on pristine PDMS and SSA 50:50 composite films (Figure 8A2 and C2, arrows). No major qualitative differences could be observed while comparing both polymeric materials. As a side note, it appears that a fewer amount of cellular aggregates were present on SSA 50:50 compared to PDMS. Also, the aggregates attached to the surfaces on SSA 50:50 appeared more flattened (Figure 8C1). As expected, treatment with air plasma improved cellular attachment and spreading on both surfaces significantly, leading to the formation of remarkable lamellipodia protrusions and a confluent monolayer (Figure 8B2 and 8D2).

Release of LDH after 3 days of culture was used as an indicator to determine cytotoxic effects (Figure 9A). In general, LDH levels were comparable for cells cultured on both polymeric materials, with less than 5\% cytotoxicity (pristine PDMS: $2.8 \pm 2.0 \%$; pristine SSA 50:50: 4.5 $\pm 3.6 \%$; plasma treated PDMS: $3.4 \pm 1.5 \%$; plasma treated SSA 50:50: $3.4 \pm 1.6 \%$ ). These results are comparable to data presented in our previously published study focusing on the investigation of both elastomers. ${ }^{8}$ To further validate the results of the LDH assay, a trypan blue exclusion test was performed. Additionally, the entire cell population was determined to display differences in proliferation activity (Figure 9B). In general less than 5\% Trypan Blue positive cells were counted (pristine PDMS: $2.4 \pm 0.3 \%$; pristine SSA 50:50: $3.8 \pm 2.5 \%$; plasma treated PDMS: $0.74 \pm 1.3 \%$ plasma treated SSA 50:50: $0.95 \pm 1.6 \%$ ). 

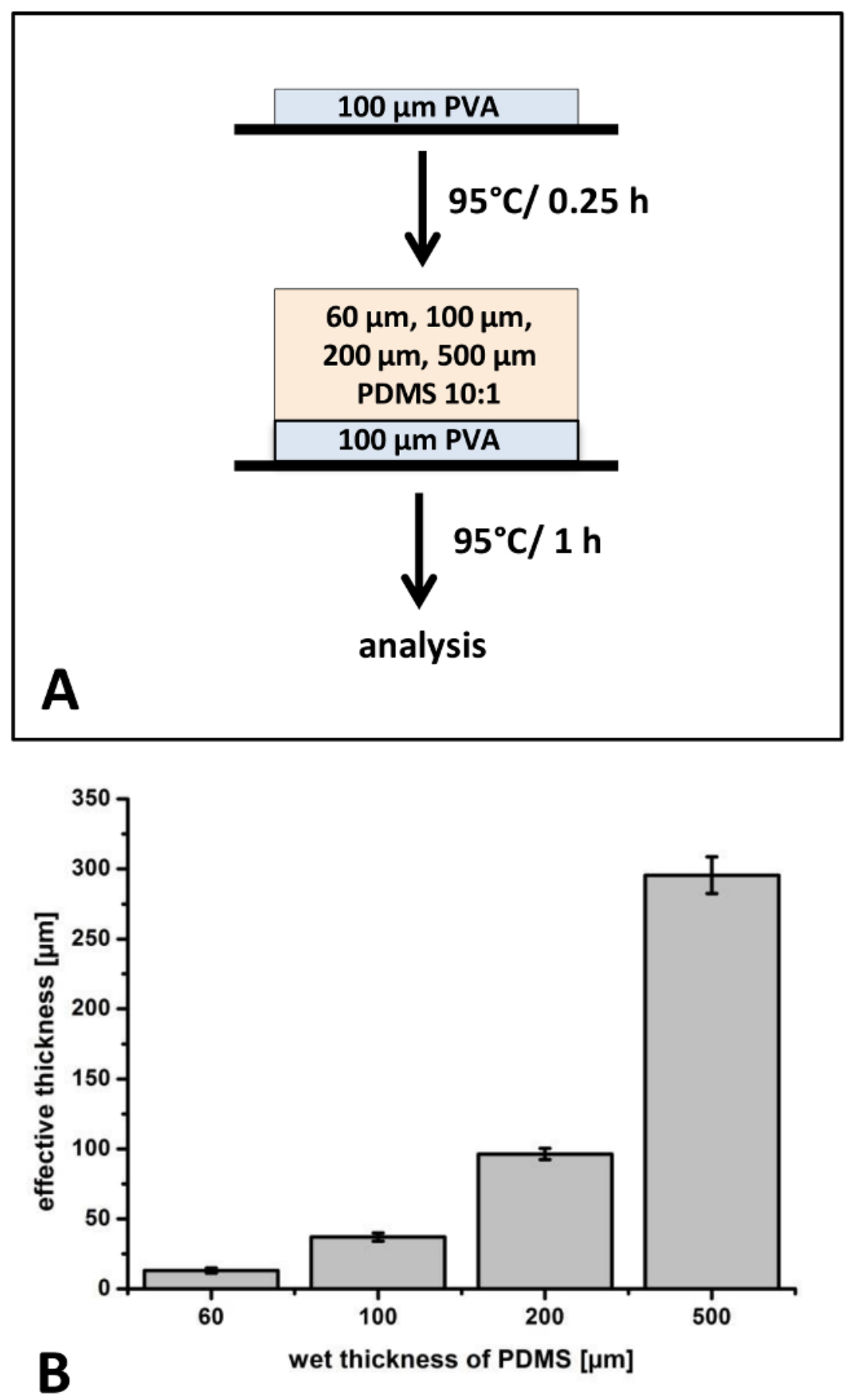

Figure 1: Preparation of PDMS films on poly-(vinyl alcohol) (PVA) coated PET foil: The process for manufacturing PDMS films with varying thickness on a PET foil was applied to determine reproducibility and handling performance (A). The thicknesses of the PDMS films were analyzed with optical microscopy after curing at $95^{\circ} \mathrm{C}(\mathrm{B}) . \mathrm{N}=3$ independently manufactured films were analyzed. From each film, three different locations were chosen, cut and 3 positions on each sample were analyzed $(k=27)$. Error bars represent standard deviation. Please click here to view a larger version of this figure. 

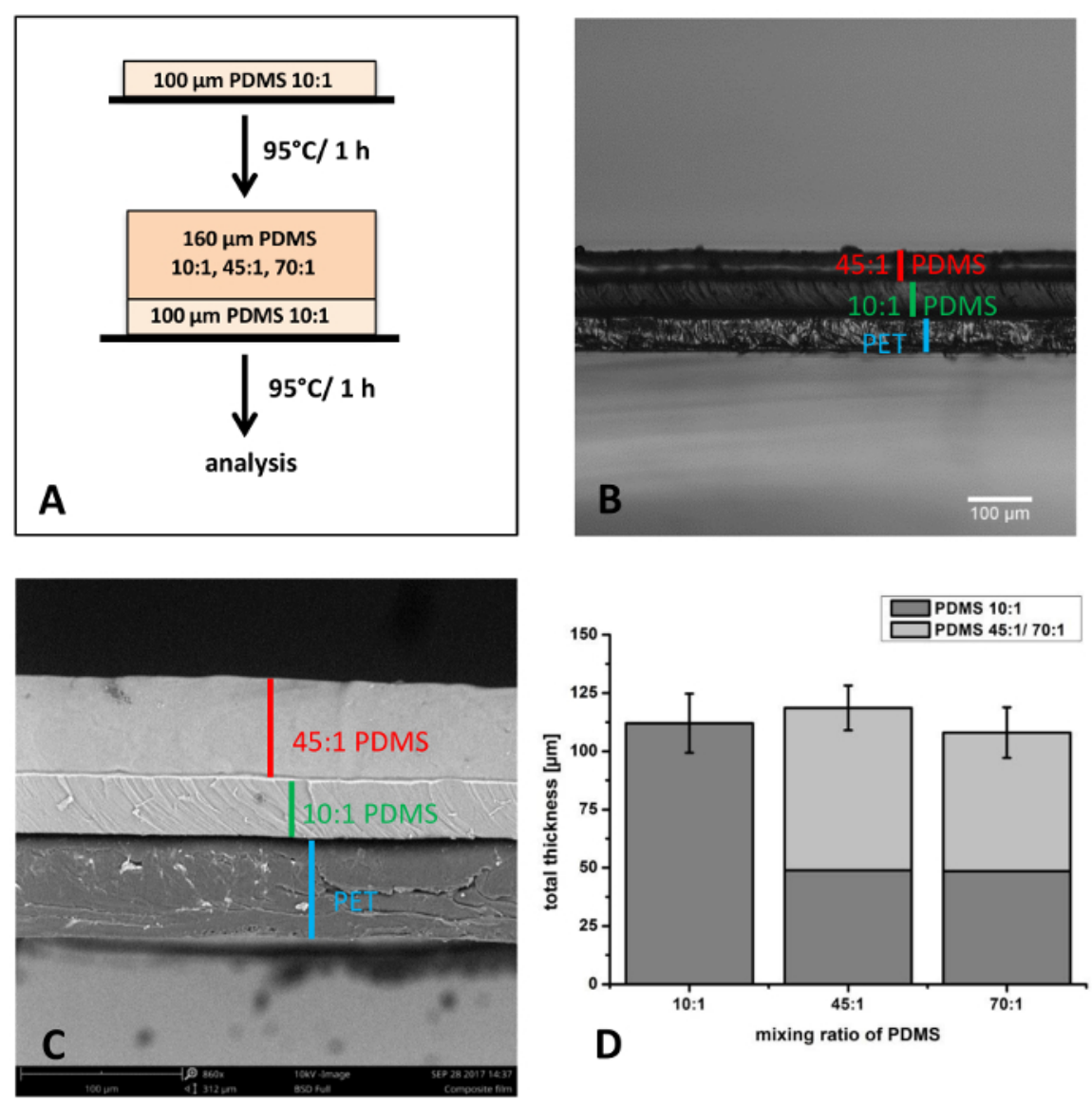

D

mixing ratio of PDMS

Figure 2: Preparation of composite films of PDMS prepared in different mixing ratios: Composite films exhibiting different mixing ratios of the base (component A) to crosslinker (component B) of PDMS were manufactured by doctor blade technique. The top layer consisting of PDMS in the ratios 10:1 (component A:B), 45:1 and 70:1 were applied on top of a previously cured PDMS 10:1 film (A). After subsequent curing at $95{ }^{\circ} \mathrm{C}$ thickness of the composite films was analyzed by optical microscopy (B) and scanning electron microscopy (C). $\mathrm{N}=3$ independent experiments were performed and analyzed with optical microscopy (D). Form each independent manufactured film, three different locations were chosen, cut and 3 positions on each samples were analyzed $(k=27)$. Error bars represent standard deviation. Please click here to view a larger version of this figure.

A1
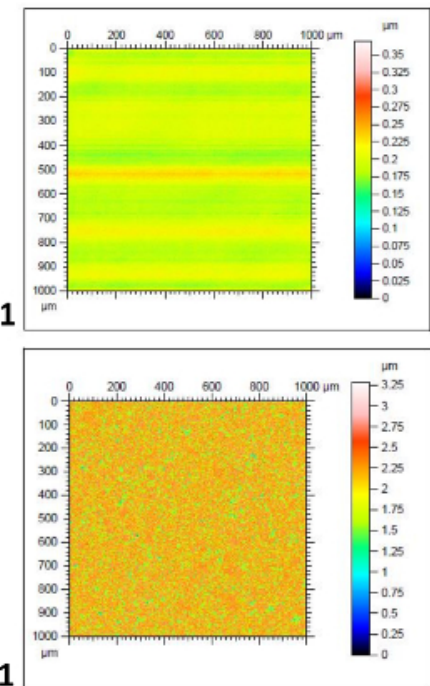

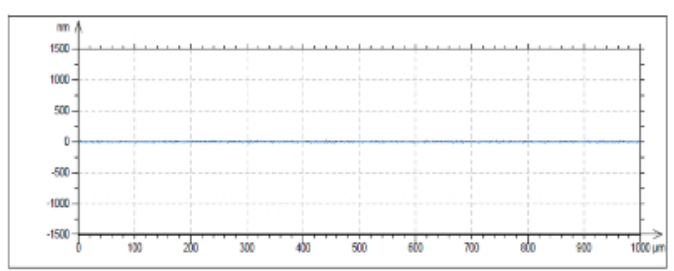

A2

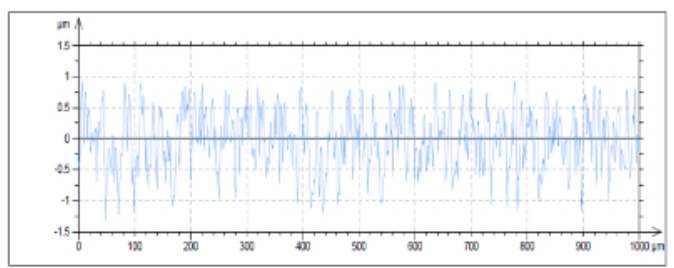

B2

Figure 3: Determination of topographical surface roughness of the two substrates used for the adhesion measurements: Two glass substrates possessing different surface roughness were characterized. Three dimensional profilometric analysis of the surface was performed on the 'smooth' substrate GS (A1) and the 'rough' substrate GR (B1). Corresponding single line curves are depicted in A2 and B2). Please click here to view a larger version of this figure. 


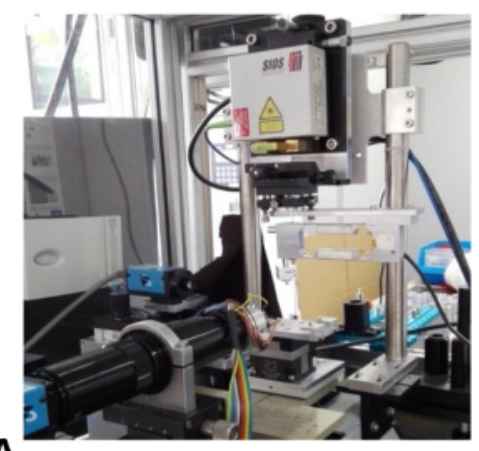

A

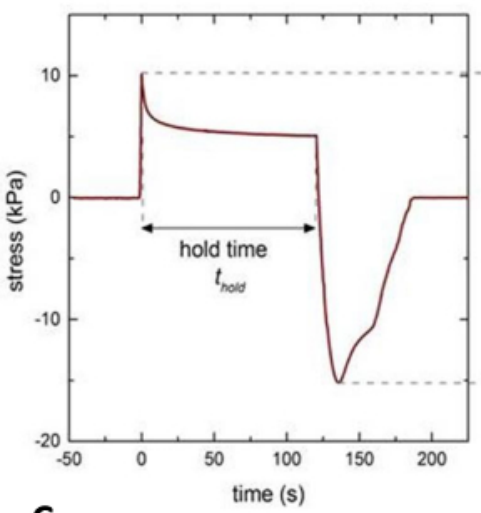

C

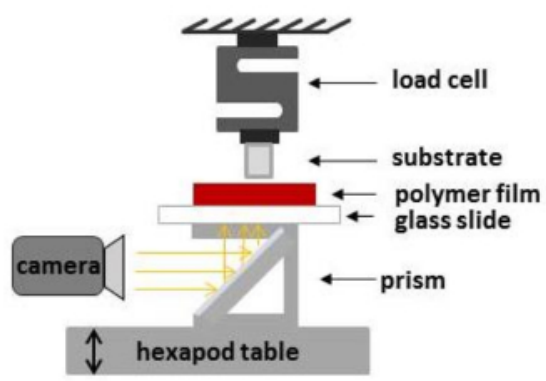

B

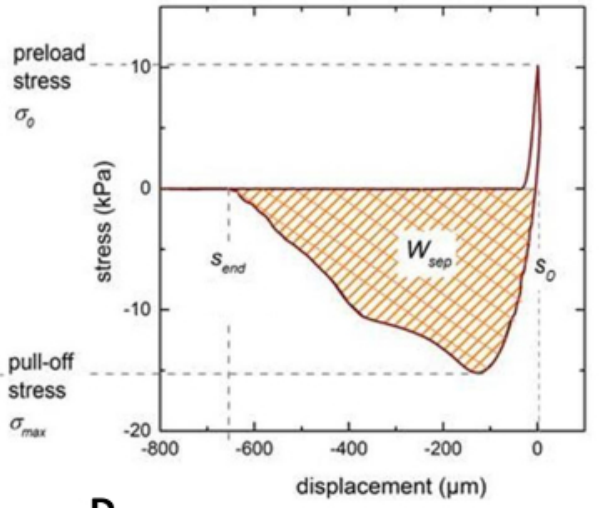

Figure 4: Principle of the normal adhesion measurements: A custom build setup was used to characterize the adhesion properties of the polymer samples. The measurement setup is depicted in (A) and details are shown in (B). For the measurement analysis, stress was determined from a stress time curve (C). Work of separation was determined by an integration of the stress- displacement curve between $\mathrm{s}_{\text {end }}$ and $\mathrm{s}_{0}(\mathbf{D})$. Please click here to view a larger version of this figure.

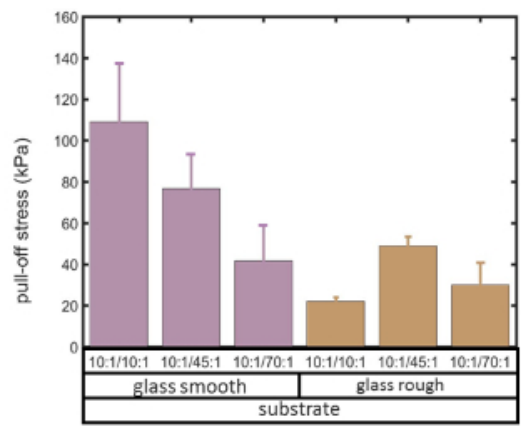

A

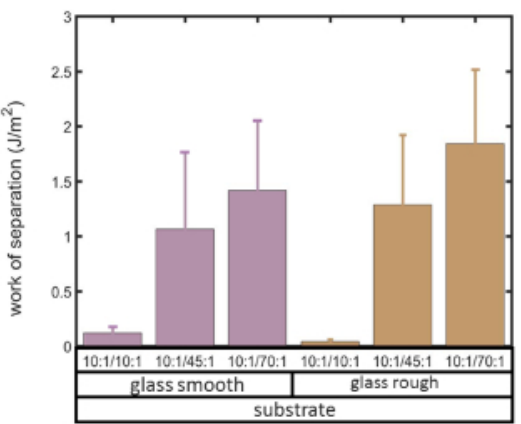

B

Figure 5: Determination of the adhesion properties of composite films with different mixing ratios of PDMS: Pull-off stress (A) and work of separation (B) of the composite films manufactured of PDMS in the mixing ratios 10:1, 45:1 and 70:1 were measured. For the analysis, a 'smooth' glass substrate (GS) exhibiting a $R_{a}=0.013 \mu \mathrm{m}$ and a 'rough' glass substrate (GR) with $R_{a}=0.338 \mu \mathrm{m}$ were used. $\mathrm{N}=3$ independently manufactured films were analyzed. From each film, two pieces were chosen and three different positions on each sample were analyzed ( $k=18)$. Error bars represent standard deviation. Please click here to view a larger version of this figure. 

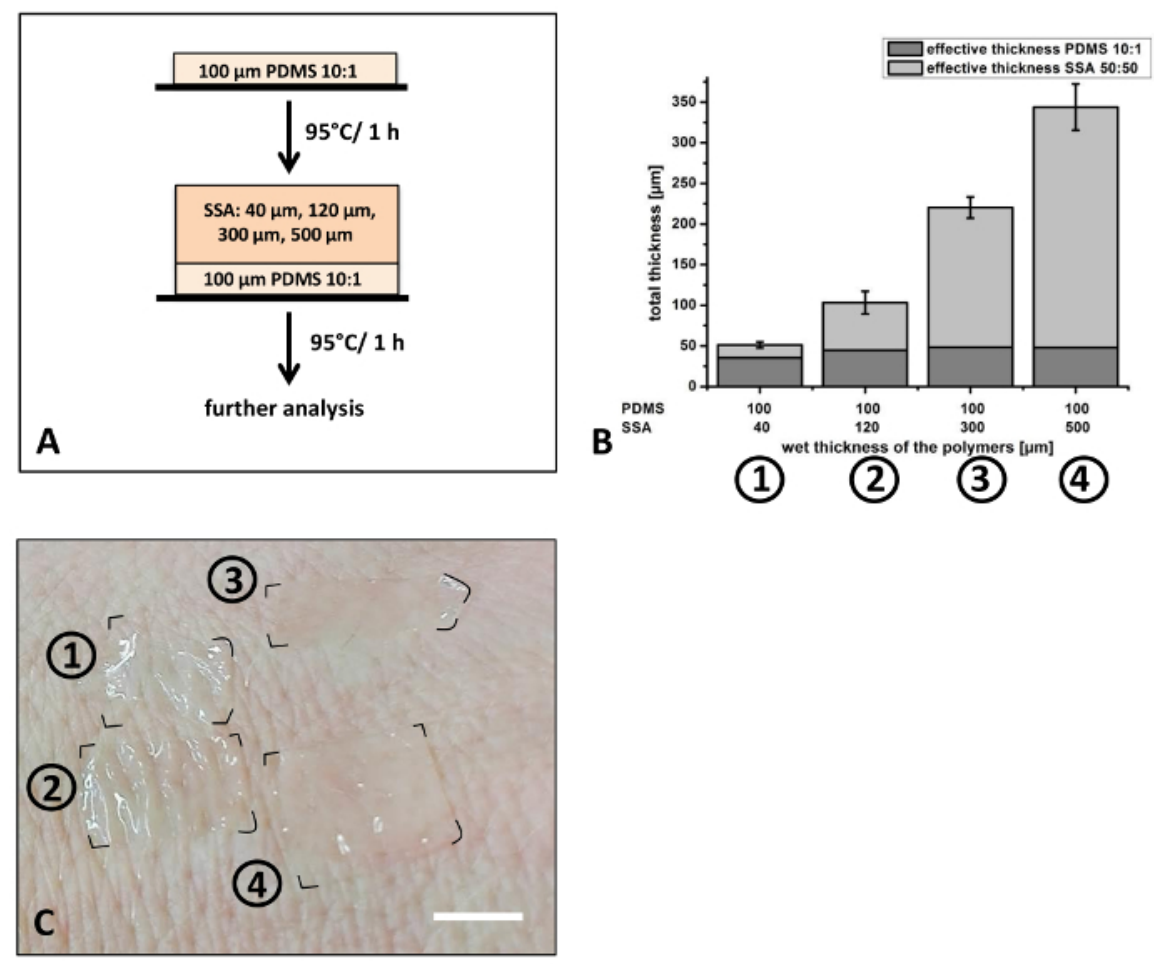

Figure 6: Preparation of composite films of SSA with varying thickness: SSA 50:50 was applied on top of a previously cured PDMS 10:1 film (A). Different wet thicknesses of this layer ranging from 40 to $500 \mu \mathrm{m}$ were applied and the thickness after curing investigated with optical microscopy (B). Attachment of the films to the back of a volunteers hand displayed that films with a total thickness of approx. $100 \mu \mathrm{m}$ (film \#2) conformed well to the roughness of the skin (C). Thickness of the single layers and the total thickness of the composite films are shown in Figure 6B. For the analysis $n=3$ independently manufactured samples were measured with optical microscopy. From each film, three different locations were chosen, cut and 3 positions on each sample were analyzed $(\mathrm{k}=27)$. Error bars represent standard deviation. Scale bar in $6 \mathrm{C}$ depicts approx. $1 \mathrm{~cm}$. Please click here to view a larger version of this figure. 


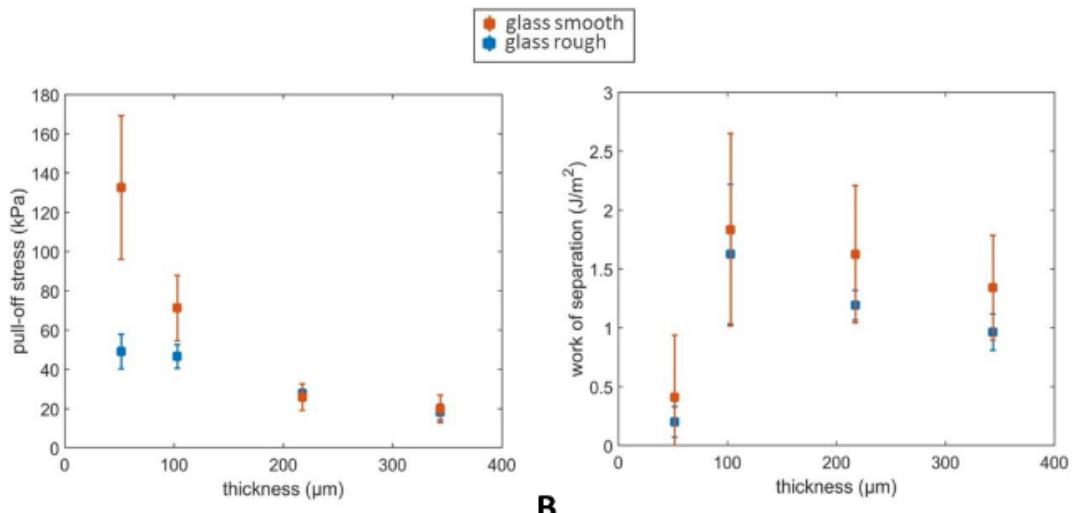

A

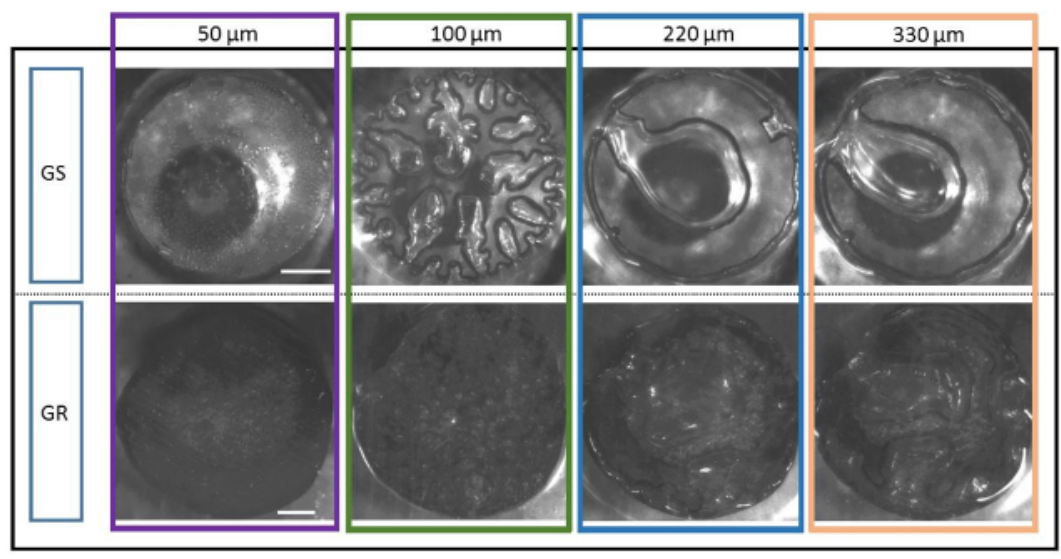

Figure 7: Determination of the adhesion properties of composite films of the soft skin adhesive: Thin composite films of SSA as a top layer and PDMS 10:1 as backing layer were manufactured. The thickness of the top layer was varied between 50 and $330 \mu \mathrm{m}$. Pull-off stress (A) and work of separation (B) of the composite films measured with two different glass substrates were analyzed (a 'smooth' glass substrate (GS) exhibiting an $R_{a}=0.013 \mu \mathrm{m}$ and a 'rough' glass substrate $(G R)$ with $R_{a}=0.338 \mu \mathrm{m}$ ). Exemplary pictures of the detachment mechanisms are visualized in $\mathbf{C}$. For data analysis $n=3$ independently manufactured experiments were analyzed. From each film, two pieces were chosen and three different positions on each sample were analyzed $(\mathrm{k}=18)$. Error bars represent standard deviation. Scale bars in $7 \mathrm{C}$ depict $0.5 \mathrm{~mm}$. Please click here to view a larger version of this figure. 


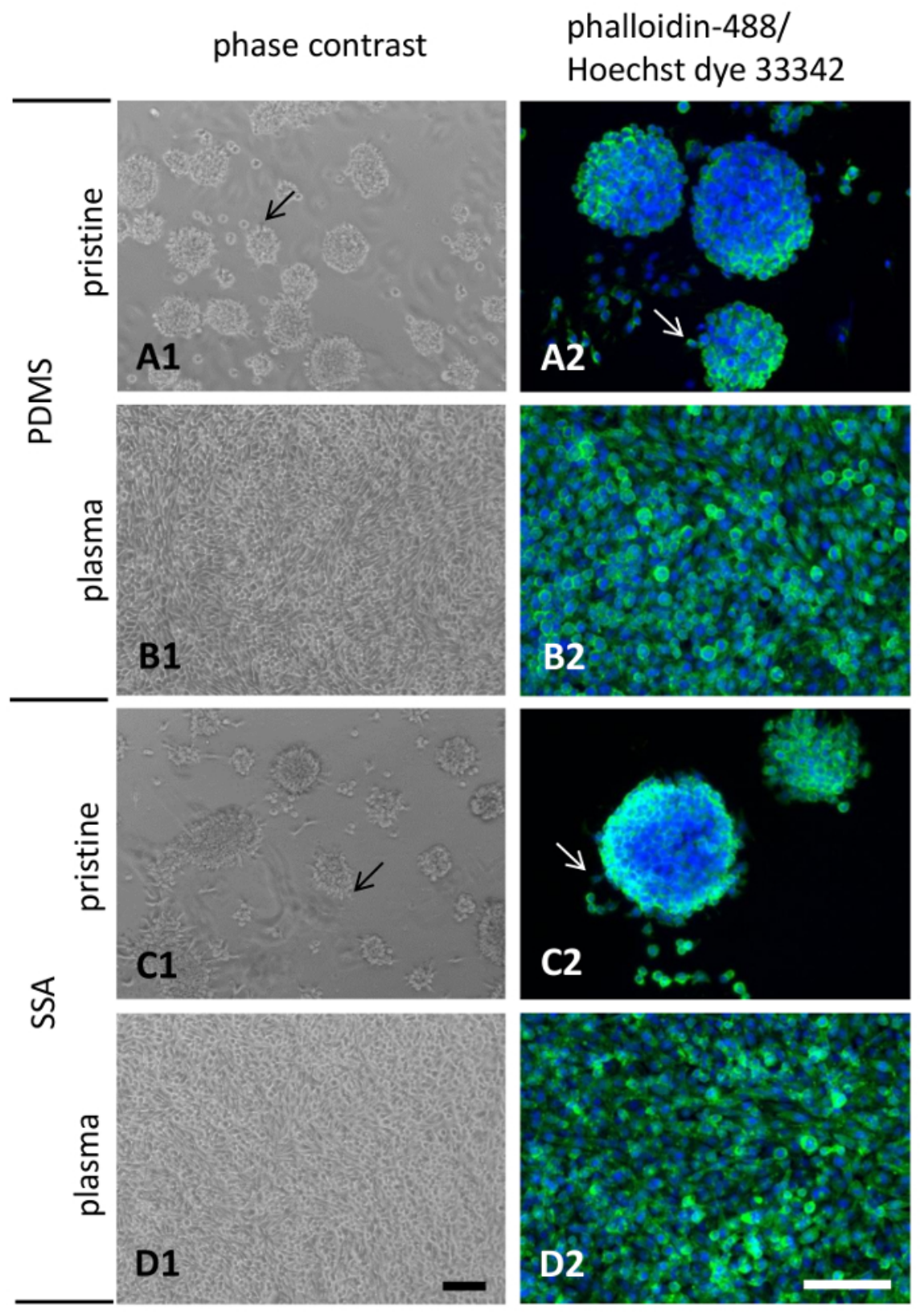

Figure 8: Cellular morphology of L929 fibroblasts cultured on thin films: L929 murine fibroblasts were cultured for 3 days on the thin films manufactured from PDMS (A1, A2, B1, B2) or SSA (C1, C2, D1, D2). To increase hydrophilicity of the surfaces air plasma treatment was performed (B1, B2, D1, D2). Scale bars in D1 and D2 depict $100 \mu \mathrm{m}$. Please click here to view a larger version of this figure. 


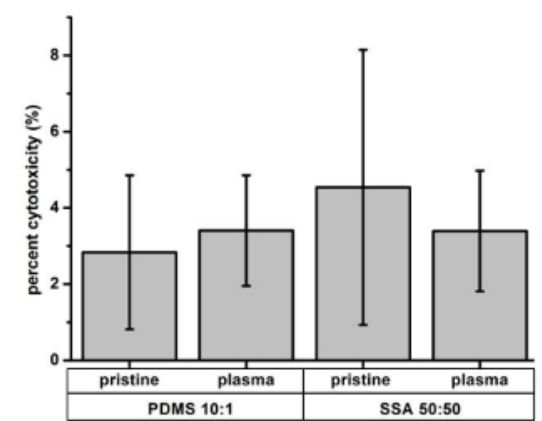

A

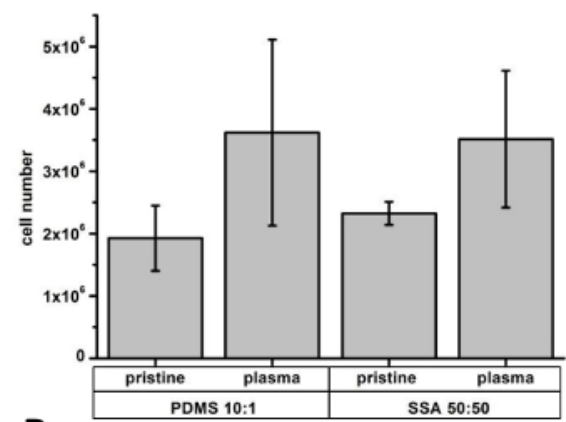

B

Figure 9: Determination of cytotoxicity and cellular proliferation: For the determination of cytotoxic effects and cellular proliferation, L929 cells were seeded for three days on PDMS 10:1 and SSA 50:50 composite films. Release of lactate dehydrogenase (LDH) was determined by an LDH activity assay and revealed less than $5 \%$ cytotoxicity (A). Total cell number after the cultivation period was assessed after manual counting the single cells with a Neubauer chamber (B). $N=3$ independently performed experiments were analyzed. Error bars represent standard deviation. Please click here to view a larger version of this figure.

\section{Discussion}

The design of composite structures enables the simple adjustment of material properties, such as Young's modulus or the thickness of the samples. The Young's modulus of PDMS can be effectively changed in a wide range by either altering the mixing ratio between the two components or manufacturing of blends using a different silicone elastomer ${ }^{30,31}$. The described methods are not limited to the PDMS's used in the current investigation, but especially the adhesive performance depends strongly on the specific type used. A critical step within this protocol is the manufacturing process of the composite films (Figure 1). It was shown that thickness of the films affects significantly the adhesion behavior of the films on different substrates, including skin (Figure 5 and Figure $\mathbf{6}$ ). In addition to the film thickness, time and temperature during the curing process affects the material properties ${ }^{32}$. Therefore, parameters as the thickness of the polymeric layers have to be carefully adapted and verified.

Analysis of the adhesive properties of the thin films was performed with normal force adhesion measurements using two glass substrates with different surface roughness up to $\mathrm{Ra}=0.338 \mu \mathrm{m}$ (Figure 3 ). In general, roughness impacts significantly the adhesion of surfaces, especially of elastic materials ${ }^{33,34}$. The roughness of glass can be easily varied by grinding with sandpaper of different asperity sizes, therefore allowing the fabrication of substrates exhibiting higher roughness values ${ }^{21}$. In addition, other materials, for example epoxy resin can be used for the production of substrates ${ }^{15,35}$. This might be an important modification strategy of the presented protocol. For example, if substrates exhibiting different surface free energies are needed or specific topographies are required. Here, pull-off stress and work of separation of the thin films manufactured of PDMS and SSA were analyzed with a custom-built setup (macroscopic adhesion measurement device (MAD, Figure 4)). ${ }^{36}$ Optical alignment of substrate and indenter is a critical step for the analysis of the measurement results. Therefore, adjustment of the tilt angle needs to be performed with the goniometer, as precise as possible. This can be achieved with sufficient precision by manually bringing the substrate into contact with the film surface until a horizontal contact is achieved.

In the current protocol the hold time was kept constant at one second (Figure $\mathbf{5}$ and Figure 7). Especially for the investigation of the adhesive performance of an elastic film to a rough substrate surface, an extension of the hold time provides additional information. For example, an increase in pull-off stress with increasing hold time has been reported ${ }^{8}$. In addition to the measurements performed in the current protocol, other methods, for example peel tests could be performed, allowing a more comprehensive investigation of adhesion performance ${ }^{37}$.

The adhesive properties of composite films exhibiting different film thicknesses of the soft skin adhesive were determined (Figure 7). Our results are in line with published data, showing that a decrease of film thickness lead to an increase of the pull-off stress as the confinement, i.e., the ratio between substrate diameter and film thickness, increase ${ }^{38,39}$. Based on these results and the data depicted in Figure 7 , we conclude that composite films with a total thickness of approx. $100 \mu \mathrm{m}$ (a thickness of the SSA layer of approx. $60 \mu \mathrm{m}$ applied to a PDMS film with a thickness of approx. $40 \mu \mathrm{m}$ ) exhibit favorable adhesion properties on rough surfaces.

Next, experiments related to the biological characterization have been performed on pristine composite films and plasma treated composite films (Figure 8). Plasma treatment of silicon elastomers is an often applied, versatile technique to increase the hydrophilic properties of surfaces and promoting cellular attachment and cellular spreading ${ }^{40,41}$. Silicones are well known for their low toxicity and high biostability but may contain residual monomers or catalysts which might influence physiological processes, leading also to cytotoxicity ${ }^{42,43}$. In the conducted experiments we have observed less than $5 \%$ cytotoxicity using LDH release as an indicator and a Trypan Blue exclusion assay. In the presented protocol, the entire cellular population, including cellular aggregates detached form the surface has been analyzed for proliferation analysis (Figure 9B). A modification of the protocol could produce more differentiated results. For each sample, the supernatant containing detached cellular aggregates could be transferred to a separate reaction tube and not combined with the cells enzymatically removed from the polymer surface. This would allow the exact assessment of cells attached to the surface and eventually reveal a more detailed determination of the influence of the polymers on the cellular adhesion process. In addition to the immunocytochemical methods presented here, cells might be harvested for investigation with immunoblot methods, allowing a detailed quantitative assessment of protein expression.

In summary, we have established manufacturing conditions for the production of thin elastomeric composite films for applications in advanced cell culture research. Additionally, these thin films possess high adaptability to skin roughness, enabling sophisticated design of skin adhesives. 


\section{Disclosures}

The authors have nothing to disclose.

\section{Acknowledgements}

Martin Danner is acknowledged for his assistance in preparing samples and establishment of cell culture procedures. The authors would like to thank Biesterfeld Spezialchemie GmbH (Hamburg, Germany), especially Robert Radsziwill for continuous support and discussions. The research leading to these results has received funding from the European Research Council under the European Union's Seventh Framework Programme (FP/2007-2013) /ERC Grant Agreement n. 340929.

\section{References}

1. Lloyd, A. W., Faragher, R. G. A., Denyer, S. P. Ocular biomaterials and implants. Biomaterials. 22, 769-785 (2001).

2. Zhang, M., Wu, J., Wang, L., Xiao, K., Wen, W. A simple method for fabricating multi-layer PDMS structures for 3D microfluidic chips. Lab Chip. 10, 1199-1203 (2010).

3. Kwak, M. K., Jeong, H. E., Suh, K. Y. Rational design and enhanced biocompatibility of a dry adhesive medical skin patch. Adv. Mater. 23 , 3949-3953 (2011).

4. Gun Park, D., Chul Shin, S., Won Kang, S., Tae Kim, Y. Development of flexible self adhesive patch for professional heat stress monitoring service. Conf. Proc. IEEE Eng. Med. Biol. Soc. 4, 3789-3792 (2005).

5. Thomas, X. Silicone Adhesives in Healthcare Applications. Dow corning Lit. (2013).

6. Fuard, D., Tzvetkova-Chevolleau, T., Decossas, S., Tracqui, P., Schiavone, P. Optimization of poly-di-methyl-siloxane (PDMS) substrates for studying cellular adhesion and motility. Microelectron. Eng. 85, 1289-1293 (2008).

7. Wang, Z., Volinsky, A. A., Gallant, N. D. Crosslinking effect on polydimethylsiloxane elastic modulus measured by custom-built compression instrument. J. Appl. Polym. Sci. 131, 41050 (2014).

8. Fischer, S.C.FL., Kruttwig, K., Bandmann, V., Hensel, R., Arzt, E. Adhesion and cellular compatibility of silicone-based skin adhesives. Macromol. Mater. Eng. 1-11 (2017).

9. Martina, D., Creton, C., Damman, P., Jeusette, M., Lindner, A. Adhesion of soft viscoelastic adhesives on periodic rough surfaces. Soft Matter. 8, 5350-5357 (2012).

10. Brown, X. Q., Ookawa, K., Wong, J. Y. Evaluation of polydimethylsiloxane scaffolds with physiologically-relevant elastic moduli: Interplay of substrate mechanics and surface chemistry effects on vascular smooth muscle cell response. Biomaterials. 26, $3123-3129$ (2005).

11. Song, F., Ren, D. Stiffness of cross-linked poly(dimethylsiloxane) affects bacterial adhesion and antibiotic susceptibility of attached cells. Langmuir. 30, 10354-10362 (2014).

12. Buxboim, A., Rajagopal, K., Brown, A. E. X., Discher, D. E. How deeply cells feel: methods for thin gels. J. Phys. Condens. Matter. 22, 194116 (2010)

13. Discher, D. E., Janmey, P., Wang, Y.-L. Tissue cells feel and respond to the stiffness of their substrate. Science. 310, 1139-43 (2005).

14. Engler, A. J., Sen, S., Sweeney, H. L., Discher, D. E. Matrix Elasticity Directs Stem Cell Lineage Specification. Cell. 126, 677-689 (2006).

15. Roth, J. et al. Surface functionalization of silicone rubber for permanent adhesion improvement. Langmuir. 24, 12603-12611 (2008).

16. Thiébaud, P., Lauer, L., Knoll, W., Offenhäusser, A. PDMS device for patterned application of microfluids to neuronal cells arranged by microcontact printing. Biosens. Bioelectron. 17, 87-93 (2002).

17. Tourovskaia, A., Figueroa-Masot, X., Folch, A. Differentiation-on-a-chip: a microfluidic platform for long-term cell culture studies. Lab Chip. 5, 14-9 (2005).

18. Peterson, S. L., McDonald, A., Gourley, P. L., Sasaki, D. Y. Poly(dimethylsiloxane) thin films as biocompatible coatings for microfluidic devices: Cell culture and flow studies with glial cells. J. Biomed. Mater. Res. - Part A. 72, 10-18 (2005).

19. Wan, Y. et al. Nanotextured substrates with immobilized aptamers for cancer cell isolation and cytology. Cancer. 118, 1145-1154 (2012).

20. Ross, A. M., Jiang, Z., Bastmeyer, M., Lahann, J. Physical aspects of cell culture substrates: Topography, roughness, and elasticity. Small. 8, 336-355 (2012).

21. Barreau, V. et al. Fibrillar Elastomeric Micropatterns Create Tunable Adhesion Even to Rough Surfaces. Adv. Funct. Mater. 26, 4687-4694 (2016).

22. Briggs, G. A. D., Briscoe, B. J. The effect of surface topography on the adhesion of elastic solids. J. Phys. D. Appl. Phys. 10, 2453-2466 (1977).

23. Dapp, W. B., Lücke, A., Persson, B. N. J., Müser, M. H. Self-affine elastic contacts: Percolation and leakage. Phys. Rev. Lett. 108, 1-4 (2012).

24. Putignano, C., Carbone, G., Dini, D. Mechanics of rough contacts in elastic and viscoelastic thin layers. Int. J. Solids Struct. 69 - 70, 507-517 (2015).

25. Laulicht, B., Langer, R., Karp, J. M. Quick-release medical tape. Proc. Natl. Acad. Sci. 109, 18803-18808 (2012).

26. Kim, T., Park, J., Sohn, J., Cho, D., Jeon, S. Bioinspired, Highly Stretchable, and Conductive Dry Adhesives Based on 1D-2D Hybrid Carbon Nanocomposites for All-in-One ECG Electrodes. ACS Nano. 10, 4770-4778 (2016).

27. Adamietz, I. A. et al. Effect of self-adhesive, silicone-coated polyamide net dressing on irradiated human skin. Radiat. Oncol. Investig. 2, 277-282 (1994).

28. White, R. Evidence for atraumatic soft silicone wound dressing use. Wounds UK. 1, 104-109 (2005).

29. Quan, M.B., Edwards, C., Marks, R. Non-invasive in vivo techniques to differentiate photodamage and ageing in human skin. Acta Derm. Venereol. 77, 416-419 (1997).

30. Brown, X. Q., Ookawa, K., Wong, J. Y. Evaluation of polydimethylsiloxane scaffolds with physiologically-relevant elastic moduli: Interplay of substrate mechanics and surface chemistry effects on vascular smooth muscle cell response. Biomaterials. 26, 3123-3129 (2005).

31. Palchesko, R. N., Zhang, L., Sun, Y., Feinberg, A. W. Development of Polydimethylsiloxane Substrates with Tunable Elastic Modulus to Study Cell Mechanobiology in Muscle and Nerve. PLoS One. 7, e51499 (2012). 
32. Johnston, I. D., McCluskey, D. K., Tan, C. K. L., Tracey, M. C. Mechanical characterization of bulk Sylgard 184 for microfluidics and microengineering. J. Micromechanics Microengineering. 24, 35017 (2014).

33. Persson, B. N. J., Gorb, S. The effect of surface roughness on the adhesion of elastic plates with application to biological systems. J. Chem. Phys. 119, 11437 (2003).

34. Peressadko, A. G., Hosoda, N., Persson, B. N. J. Influence of surface roughness on adhesion between elastic bodies. Phys. Rev. Lett. 95, 1-4 (2005).

35. Purtov, J. et al. Measuring of the hardly measurable: adhesion properties of anti-adhesive surfaces. Appl Phys A. 111, 183-189 (2013).

36. Kroner, E., Blau, J., Arzt, E. Note: An adhesion measurement setup for bioinspired fibrillar surfaces using flat probes. Rev. Sci. Instrum. 83, 16101 (2012).

37. Sun, S., Li, M., Liu, A. A review on mechanical properties of pressure sensitive adhesives. Int. J. Adhes. Adhes. 41, 98-106 (2013).

38. Webber, R. E., Shull, K. R., Roos, A., Creton, C. Effects of geometric confinement on the adhesive debonding of soft elastic solids. Phys. Rev. E. 68, 21805 (2003).

39. Creton, C., Lakrout, H. Micromechanics of flat-probe adhesion tests of soft viscoelastic polymer films. J. Polym. Sci. Part B Polym. Phys. 38, 965-979 (2000).

40. Tan, S. H., Nguyen, N.-T., Chua, Y. C., Kang, T. G. Oxygen plasma treatment for reducing hydrophobicity of a sealed polydimethylsiloxane microchannel. Biomicrofluidics. 4, 32204 (2010).

41. Kim, B., Peterson, E.T.K., Papautsky, I. Long-term stability of plasma oxidized PDMS surfaces. Conf. Proc. IEEE Eng. Med. Biol. Soc. 7, 5013-5016 (2004).

42. Briganti, E. et al. Silicone based polyurethane materials: A promising biocompatible elastomeric formulation for cardiovascular applications. J. Mater. Sci. Mater. Med. 17, 259-266 (2006).

43. Regehr, K. J. et al. Biological implications of polydimethylsiloxane-based microfluidic cell culture. Lab Chip. 9, 2132 (2009). 